\title{
Discurso de futuros docentes acerca de la diversidad intercultural*
}

\section{Concepción Carrasco Carpio}

Universidad de Alcalá. Facultad de Ciencias Económicas, Empresariales y Turismo.

Departamento de Economía. Área de Sociología

concha.carrasco@uah.es

\section{Resumen}

El discurso teórico sobre la diversidad intercultural se encuentra todavía muy alejado de la práctica. Una de las causas de esta distancia es la falta de formación de los docentes (presentes y futuros). Para poner en práctica el enfoque intercultural, resulta imprescindible conocer el discurso de los agentes que lo aplicarán. El objetivo de este análisis es doble: por un lado, conocer la perspectiva dominante del discurso intercultural en un estudio de caso con varias subunidades de futuros docentes y, por otro lado, determinar qué barreras identifican en relación con las prácticas interculturales en las escuelas.

Para ello, se crearon 30 grupos de discusión entre el alumnado (302 estudiantes) de primer curso de grado en Educación Infantil y de grado en Educación Primaria de la Facultad de Educación de la Universidad de Alcalá (curso académico 2011-2012). Los resultados indican que, tal vez debido a la escasez de experiencia en diversidad intercultural, la principal perspectiva del discurso es compensatoria y asimilacionista, $y$, además, que hay cuatro barreras principales entre la teoría y la práctica: la escasez de conocimientos, la inconsistencia de los valores personales con la interculturalidad, las ideas estereotipadas y la falta de una visión transversal en el currículum.

Palabras clave: formación inicial; educación intercultural; estereotipos; diversidad intercultural; formación de profesores; multiculturalismo.

* Quiero mostrar mi agradecimiento al Dr. Juan Miguel Fernández-Balboa, por sus sabias aportaciones y recomendaciones; a la profesora Ana María Ayuso Álvarez, por su participación en el trabajo de campo, y, finalmente, al alumnado, por su colaboración. 
Abstract. Discourse of future teachers about intercultural diversity

The theoretical discourse on intercultural diversity is still far removed from practice. One reason for this gap is the lack of properly trained teachers (both present and future). To implement the intercultural approach, it is essential to know the discourse of the agents who will apply it. The purpose of this study is twofold: (1) to determine the dominant approach to intercultural discourse through a case study with subcases of future teachers, and (2) to determine what barriers they identify regarding the implementation of intercultural practices in schools. To this effect, 30 focus groups consisting of first-year students (302 in total) enrolled in the BA in Early Childhood and Elementary Education at the Faculty of Education at the University of Alcalá (academic year 2011-2012) were interviewed. The results indicate that (a) perhaps due to a lack of experience in cultural diversity, the primary discourse perspective is compensatory and assimilationist; and (b) there are four main barriers between theory and practice: lack of knowledge, personal values inconsistent with interculturality, stereotyped ideas, and the lack of a transversal vision in the curriculum.

Keywords: initial training; intercultural education; stereotypes; intercultural diversity; teacher training; multiculturalism.

\section{Sumario}
1. Contexto de diversidad intercultural
4. Conclusiones
2. Método
Referencias bibliográficas

3. Resultados

\section{Contexto de diversidad intercultural}

El sistema educativo español ha visto como, en el horizonte temporal 20012013, el número de alumnos extranjeros que han accedido a las distintas etapas académicas se ha cuadruplicado, puesto que ha pasado de 207.112 a 755.156 (un $9,1 \%$ del total de alumnado en enseñanzas de régimen general no universitario) en el curso escolar 2012-2013 (Ministerio de Educación, Cultura y Deporte, 2013). Esta diversidad, asociada al fenómeno migratorio, plantea un reto en la formación de los futuros profesionales de la docencia, ya que han de poner en práctica nuevas metodologías pedagógicas y materiales didácticos, asi como conocer los mecanismos existentes para obtener el mayor rendimiento posible de la diversidad. Saber gestionar esta diversidad es un factor clave para el buen funcionamiento del aula, los resultados educativos y la integración. En palabras de Terrén (2003: 263): «[...] la capacidad de integración de una sociedad es directamente proporcional a la diversidad compatible que es capaz de albergar", a lo que se puede añadir: y a la capacidad de gestionarla. Los centros educativos albergan muchas diversidades (no solo la relativa a las distintas nacionalidades), y la responsabilidad de su gestión recae, en gran medida, en los docentes. 
Desde los años noventa, se ha ido imponiendo el término diversidad intercultural (especialmente en Europa) con referencia específica a las minorías étnicas, pero, ¿qué se esconde detrás de este concepto en el ámbito educativo?

Los enfoques interculturales basados en el paradigma de la simetría cultural se han ido imponiendo en el debate académico como los más apropiados para abordar la diversidad cultural (Aguado et al., 2008; Besalú, 2012; Merino y Muñoz, 1995; Muñoz, 1997, 2001; Santos, 1994), especialmente los modelos holístico y sociocrítico, frente al enfoque asimilacionista sustentado sobre el paradigma de la desigualdad y los enfoques que potencian las diferencias culturales, basados en el paradigma de la diferencia.

El modelo holístico trata de implicar a toda la institución escolar en la educación intercultural, de manera que lo «intercultural» no sea un adjetivo, sino un "todo» que impregne al sistema educativo (Banks, 1989, 1991, 1996; Nieto y Santos, 1997; Osuna, 2012). De otra parte, el modelo sociocrítico promueve la construcción de una sociedad más justa, en lucha contra la asimetría cultural, económica, social y política (Alegret, 1992; Muñoz, 1997). Estos dos modelos se analizan en la literatura como un avance con respecto al paradigma asimilacionista con el que se comenzó a abordar la educación intercultural, modelo centrado en políticas educativas de carácter compensatorio (Bereiter y Engelman, 1966; Jensen, 1969).

Las deficiencias del modelo asimilacionista y, por extensión, de las políticas educativas compensatorias han sido puestas en evidencia por numerosos informes e investigaciones (Bernstein, 1989; Dietz y Mateos, 2011; Giroux, 1992; Merino y Muñoz, 1995), entre los que destaca la atribución al ambiente familiar, cultural y social de la falta de rendimiento del alumnado. Bajo esta perspectiva, se asigna la causa del déficit a dicho ambiente, el cual debe ser compensado con programas y medidas específicas en las aulas escolares. Para Bernstein (1989), la educación compensatoria centra la atención en la familia y los niños (a quienes hace responsables del fracaso escolar) y olvida la importancia del contexto pedagógico. Por su parte, los enfoques que potencian las diferencias culturales acaban promoviendo la segregación como alternativa docente, con el objetivo de preservar las identidades étnicas y culturales (las aulas segregadas sería un ejemplo de este modelo).

En España, el principal discurso intercultural en el ámbito educativo potencia la visión de la inmigración como enriquecimiento cultural, pero, a la vez, apunta a un amplio repertorio de problemas a resolver: idiomas diferentes, entrada cuando el curso ya está iniciado, carencias curriculares, falta de recursos, entre otros (Colectivo IOE, 2007). En muchas ocasiones, esto se traduce en la búsqueda, por parte del profesorado, de «recetas» con un marcado carácter compensatorio $\mathrm{y}$, en ocasiones, respuestas segregadoras (Baquedano-López et al., 2013). Estas preocupaciones vuelven a traer a primer plano al paradigma asimilacionista y compensatorio, enfoque que se creía superado tras la reflexión y las experiencias educativas de los últimos 15 años en España. 
En este contexto, parece necesario preguntarse: ¿por qué resulta tan difícil poner en práctica enfoques holísticos y sociocríticos como paradigmas apropiados para gestionar la diversidad intercultural? Y, en este sentido, reflexionar sobre la formación de los futuros profesionales de la enseñanza, ya que la oferta académica para los docentes en el ámbito de la interculturalidad es todavía escasa, aunque ha aumentado, especialmente en la formación permanente. Se puede considerar que la transformación de las prácticas que fomentan la interculturalidad sigue constituyendo un reto. En palabras de Garreta y Llevot (2003: 170), «la escasa formación inicial de los docentes y la no muy exitosa permanente, sigue alimentando la fractura existente entre discurso y práctica». Por su parte, Jordán (1995), en un estudio realizado en Cataluña, pone de manifiesto que la teoría va por delante de la práctica, debido a la falta de formación rigurosa y sistemática del profesorado. De hecho, los docentes siguen manifestando que necesitan adquirir competencias interculturales (Aguado et al., 2008; Martínez y Zurita, 2011).

Entre las iniciativas de formación intercultural más destacadas en el contexto español, refiriéndose a la Guía INTER (Aguado et al., 2006; Aguado et al., 2008: 287), señalan claramente algunos de los principales retos del enfoque intercultural:

Desde la perspectiva intercultural, la Guía INTER desafía los objetivos actualmente en vigor de la educación, la perspectiva de la homogeneidad, las ideas de éxito y fracaso escolar, la idea de que la educación se basa en la transmisión del conocimiento, la asociación de la diversidad cultural con determinadas categorías sociales (inmigrantes, grupos étnicos, minorías, nacionalidades), la idea de que la Educación Intercultural consiste únicamente en la celebración festiva de la diversidad, la educación compensatoria como una estrategia de adaptación/integración, la idea de que la Educación Intercultural consiste en elaborar recetas para resolver problemas específicos o que la Educación debe evaluar únicamente los resultados académicos de los estudiantes.

Resulta, por tanto, necesario profundizar en la formación inicial de los futuros profesionales de la enseñanza, así como potenciar la reflexión guiada sobre conceptos, actitudes y prácticas a este respecto (López e Hinojosa, 2012). Para poner en práctica los modelos emergentes, en consonancia con el enfoque intercultural, resulta lógico conocer las perspectivas (el discurso) de los agentes que los aplicarán. Este es precisamente el foco del presente estudio.

\section{Método}

\subsection{Participantes}

La investigación se llevó a cabo en la Facultad de Educación de la Universidad de Alcalá, con la totalidad (302) de los alumnos y las alumnas del primer curso de grado en Educación Infantil y Primaria (año académico 2011-2012). 
El perfil de los participantes se caracterizó por la juventud (un $90 \%$ tenía una edad comprendida entre 18 y 24 años), una sobrerrepresentación de mujeres (un $77,7 \%$, frente a un $23,3 \%$ de hombres) y la residencia localizada en el eje situado entre la "zona Este de Madrid» y "Guadalajara»: Guadalajara (un 19,3\%), Madrid (un 16,3\%), Alcalá de Henares (un 14,8\%), Coslada (un 8,7\%), Torrejón de Ardoz (un 8,3\%), Azuqueca de Henares (un 3,8\%) y el resto (un $28,8 \%$ ) residente en localidades más pequeñas. Además, un $91,7 \%$ estaba soltero y un 2,3\% tenía nacionalidad extranjera.

\subsection{Objetivos}

Los objetivos de este estudio fueron:

a) Conocer la perspectiva dominante en el discurso de futuros y futuras docentes en relación con la diversidad intercultural, por medio de un estudio de caso con varias subunidades de futuros docentes (Yin, 1989).

b) Dentro de dicho discurso, determinar las barreras que distancian la teoría de la práctica (Aguado et al., 2008; Garreta y Llevot, 2003; Jordan, 1995).

\subsection{Procedimiento}

Durante el curso académico 2011-2012, se crearon 30 grupos de discusión (subunidades de futuros docentes) sobre el tema de la educación intercultural. La formación de los grupos se realizó siguiendo el criterio de la homogeneidad del alumnado: edad similar (todos los grupos), composición solo de mujeres (mitad de los grupos de discusión), procedencias geográficas similares (en todos los grupos). Estos grupos de discusión fueron grabados en audio, transcritos y analizados. El material utilizado consistió en un total de 1.500 páginas de texto transcritas derivadas de 34 horas de grabación en audio. Para su análisis, se utilizó la herramienta informática Atlas.ti.

Siguiendo a Strauss y Corbin (1998, citado en Andreu et al., 2007: 67-79), el análisis consistió, en primer lugar, en la reducción del texto transcrito a códigos o etiquetas, en segundo lugar, se establecieron las categorías principales o dimensiones. En aras de cumplir con los criterios de credibilidad y fiabilidad, la clasificación de las categorías ha sido validada por medio de la confrontación con expertos en investigación cualitativa. Posteriormente, se analizaron las relaciones entre las categorías, buscando las conexiones con la central, que, en este caso, coincide con la pregunta de investigación: ¿qué paradigma sobre la diversidad intercultural domina en el discurso de los futuros docentes? (Véase el gráfico 1.)

Las referencias al discurso se organizaron atendiendo a los siguientes códigos descriptivos: grupo de mañana (M) o tarde (T), grado de Infantil (I) o grado de Primaria $(\mathrm{P})$; grupos $\mathrm{A}, \mathrm{B}$ o $\mathrm{C}$, y, por último, el código de asignación numérica. 
Gráfico 1. Proceso de análisis cualitativo

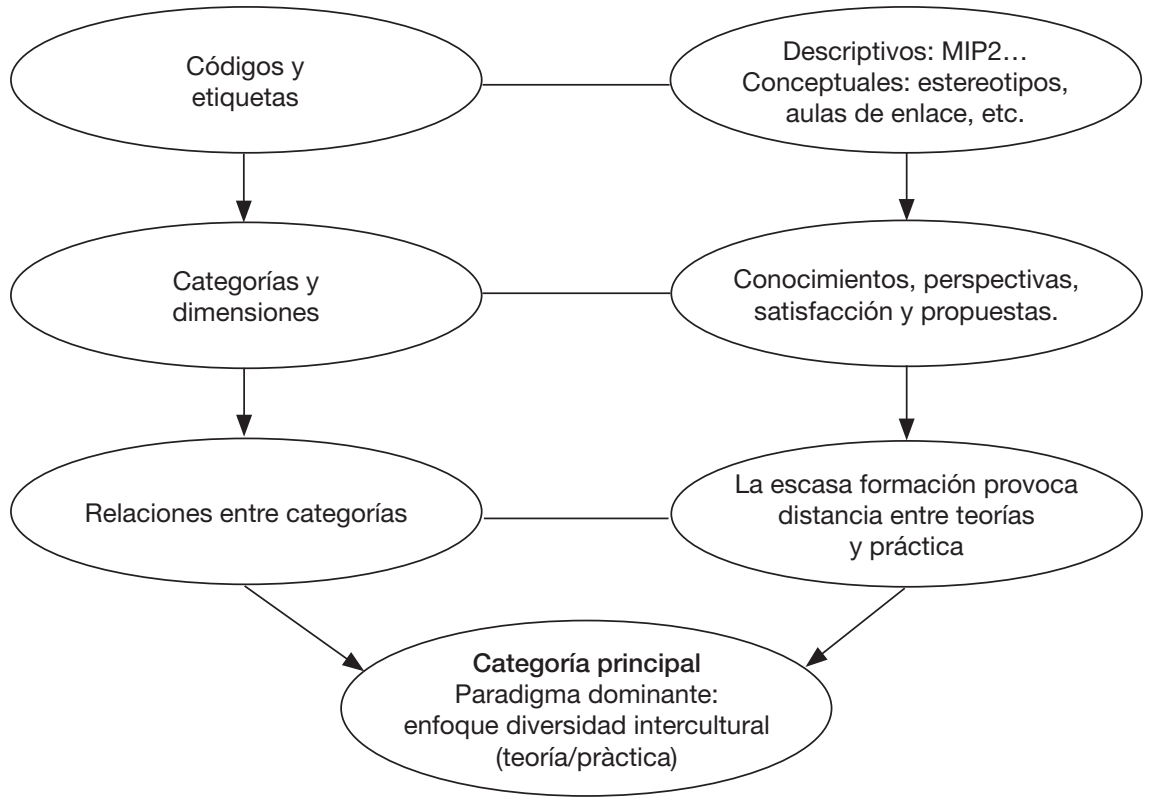

Fuente: Strauss y Corbin (1998) y elaboración propia.

\section{Resultados}

A continuación, se exponen los resultados más relevantes de este estudio en función del objetivo principal: conocer la perspectiva dominante en el discurso y, específicamente, extraer del mismo las barreras que separan a la teoría de la práctica desde un enfoque intercultural.

De los grupos de discusión realizados, se extrajeron cuatro categorías que conforman el discurso de estos futuros profesionales de la enseñanza:

a) Grado de conocimiento de la realidad intercultural en las aulas del sistema educativo español.

b) Perspectiva sobre la diversidad intercultural.

c) Grado de satisfacción con su propia formación profesional.

d) Propuestas de mejora en el sistema educativo español relacionadas con el enfoque intercultural.

\subsection{Grado de conocimiento de la realidad intercultural en las aulas del sistema educativo español}

En general, los participantes en esta investigación muestran un escaso conocimiento de la realidad intercultural presente en las aulas españolas. Ellos la 
vinculan al aumento de la inmigración y la asocian a los distintos problemas que surgen en la práctica. $\mathrm{Al}$ respecto, en uno de los grupos (MIA6), se comenta lo siguiente: "Cuando llega un niño que no sabe nada de español [...] es un problema a la hora de llevar la clase, porque no es el mismo ritmo. Tienes [sic] ciertas personas a las que tienes que darles un apoyo». Su discurso, justificándose en la teoría del déficit, se centra en las dificultades de integración del alumnado extranjero, con comentarios como el siguiente: «Yo pienso que, en algunas clases, el inmigrante que viene de fuera puede hacer que retrase a los demás, a la clase entera o a algunos alumnos; puede retrasar en temas académicos, pero supondría un enriquecimiento en el ámbito de las relaciones personales» (MIA1). Específicamente, como principal barrera, señalan el idioma:

En las prácticas, había un niño rumano. Sus padres le hablaban en rumano, sus abuelos le hablaban en rumano y solo hablaba en español cuando él iba a la escuela. La verdad es que ese niño iba muy retrasado. (MIB9)

Otro aspecto del discurso tiene que ver con las medidas que facilitan la gestión de la interculturalidad. En este sentido, destacan las aulas de enlace (nombre que reciben en la Comunidad Autónoma de Madrid ${ }^{1}$ ), las cuales están destinadas al alumnado extranjero que desconoce la lengua vehicular del nuevo lugar de residencia o que presenta un gran desfase curricular (García et al., 2010: 475). "Lo que deberíamos fomentar [los maestros] es que hubiera más apoyo, que hubiera esas aulas de enlace para que los niños [extranjeros] se pudieran integrar más fácil y más rápido» (MIA6). Al respecto, los participantes reflejan dos puntos de vista comúnmente opuestos (Del Olmo, 2012: 113). Por un lado, las aulas de enlace se relacionan con una política asimilacionista, compensatoria y segregadora:

Yo creo que llevar al niño a un aula de enlace es separatista, porque no tiene relación con los compañeros de clase, solo se relaciona con los del aula de enlace; por mucho que le intentes [sic] integrar, los demás niños lo van a ver diferente. (MPA15)

Por otro lado, se perciben como un medio facilitador de la adaptación de los recién llegados al nuevo sistema escolar y a la nueva lengua (Del Olmo, 2012; García et al., 2010; Goenechea et al., 2011; Osuna, 2012):

Lo que hacían [los maestros] era llevarlos [a los alumnos extranjeros] a un aula y allí estaban aproximadamente tres o cuatro meses [...] para que aprendieran bien el idioma, las costumbres, las características, el horario. A los tres o cuatro

1. Este tipo de medida ha recibido distintas denominaciones según preferencias de las diversas comunidades autónomas: aulas temporales de adaptación lingüistica (en Andalucía y Extremadura), aulas de acogida (en Cataluña, Islas Baleares y Murcia), aulas de inmersión lingüística (en Navarra y la Rioja) y programas de acogida al sistema educativo (en la Comunidad Valenciana), entre otras (García et al., 2010). 
meses, se integraban en el aula normal y se adaptaban de una forma mejor. (MIA5)

Otro tema emergente en el discurso de estos futuros docentes relativo a las medidas interculturales es el vinculado con las semanas culturales o con el llamado Día de la Multiculturalidad. "Trabajábamos [los alumnos] el Día de la Multiculturalidad, llevábamos comidas de distintos países» (MPA13), pero es esta la única medida que aparece, a pesar de la amplia variedad de medidas y recomendaciones existentes. Dicho de otro modo, en el discurso de los participantes, se echa en falta la referencia a recursos tan significativos como:

a) La Guía INTER (Aguado et al., 2006).

b) El Libro Blanco de la Educación Intercultural (Unión General de Trabajadores, 2010), que recoge un total de 50 propuestas de profesionales en los ámbitos de políticas educativas y lingüísticas, formación del profesorado, acciones desde el centro educativo, estrategias de centro y aula, trabajo con las familias, etc.

c) La Guía para la evaluación y mejora de la educación inclusiva (Sandoval et al., 2002, 2012), la cual representa una adaptación del índice de inclusión de Booth y Ainscow (2000) al contexto educativo español.

\subsection{Perspectiva sobre la diversidad intercultural}

Si bien, entre los participantes, hay quien aboga por un intercambio de costumbres: "la diversidad tiene que ser todos con todos: que nosotros aceptemos lo suyo, pero que ellos acepten también nuestras costumbres» (MIB7), la perspectiva dominante entre estos futuros docentes es la asimilacionista: «Es importante concienciar a las familias de que sus hijos tienen que aprender lo que se enseña en el centro educativo, adaptándose a las costumbres de nuestro país» (MPB19).

Ya no es solo que nosotros, los españoles, sepamos o no aceptar sus costumbres; yo creo que si fuera la situación al revés [...] ellos tampoco [las aceptarían]. Porque al igual que tú $[$ sic] vas a un país y tienes que aceptar todas, todas, todas sus costumbres, ellos deberían venir aquí y también aceptar todas [las nuestras] (o ser flexibles ambos). O sea, que tampoco pueden pedir algo que ellos no hacen. (MIA2)

Es más, en algunos comentarios, se detecta cierta ironía: «Lo lógico y lo que queda bien es que digamos que sí, que la interculturalidad es una oportunidad, pero, en realidad, no lo es» (MPB22).

Otro de los aspectos que emana del discurso de estos futuros maestros y maestras es que, en general, no se prepara al profesorado en términos de valores interculturales (empatía, sensibilidad, tolerancia). 
En teoría, creo que ganan los aspectos positivos; pero luego, en la práctica, ganan los negativos. No somos capaces [los profesionales de la enseñanza] de ponernos en el lugar de la otra persona. Entonces surgen los conflictos. (TIC25)

[Los profesionales de la enseñanza] no quieren o no saben cómo [llevar la teoría a la práctica]. No tienen esa experiencia y, a lo mejor, les hace falta que les enseñen o que cambien sus valores para ponerlos en práctica con los niños. (TPC28)

Tienes [sic] que modificar ciertas cosas. Hay profesores a los que les cuesta ser sensibles con el tema. Por ejemplo, con el tema de la comida, he visto, en mis prácticas, a una profesora protestar y decir: «QQué tontería esto de tener que estar cambiando la comida!». (TIC25)

Yo creo que lo que le falta a la sociedad es tolerancia. Tenemos poca tolerancia a los posibles cambios por miedo a que nos cambien. (MIB11)

Este tema está directamente relacionado con la existencia de estereotipos que, según los participantes, parecen depender de una serie de variables contextuales, principalmente: la procedencia, la religión, el nivel económico, la educación recibida en la familia y la etapa educativa.

Yo creo que no tenemos los mismos estereotipos con un inmigrante que venga de un sitio que con un inmigrante que venga de otro; tenemos ya una mentalidad de antemano $[\ldots]$. Porque no es lo mismo un rumano que un moro (o de Marruecos o un islamista). (MIA4)

Tenemos [los futuros profesionales de la enseñanza] ese prejuicio de que si es gente extranjera que tiene un nivel económico bajo se les $[s i c]$ trata peor que si fuera gente extranjera con un nivel económico alto, independientemente de que pueda ser igual que tenga más o menos dinero. (MIA1)

Muchos padres, en casa, hacen comentarios delante de sus hijos del tipo: "Me están quitando el trabajo, es que vienen aquí a [...] y se llevan todo», y esto lo escuchan los niños. (MIB10)

Yo creo que es más fácil que haya alumnos inmigrantes integrados con los demás niños españoles en las clases de educación infantil. Luego, cuando están en primaria o en secundaria, cuando ya hay unas creencias que están inculcadas, muchas veces desde casa, ya es diferente. (MIA4)

En el discurso se aprecia cierta tensión entre las ideas estereotipadas fruto de la socialización en contextos determinados, las cuales se activan automáticamente, y las adquiridas tras un proceso de reflexión crítica (Devine, 1989).

Tienes [sic] que olvidar tus ideas [estereotipadas], pero eso es la teoría, porque luego en la práctica [...]. Yo creo que, en realidad, no te olvidas de lo que piensas [los estereotipos]. Hay cosas que sí que puedes dejar a un lado, pero hay otras que salen solas. (MPA15) 
Ante esta disyuntiva, a pesar de ser conscientes de esa tensión, entre los participantes se detecta cierta impotencia en su capacidad transformadora: «Puede ser que nosotras queramos integrar a los niños; pero no porque cambiemos nosotras se va a solucionar todo este problema» (MIB11).

La sociedad ve la necesidad [de gestionar la diversidad], pero no sabe cómo hacerlo. Y, desde luego, solamente educando desde las aulas no se puede conseguir [...] La propia comunidad adulta no está abierta a ese cambio, por mucho que nos empeñemos desde las aulas. (MPA14)

\subsection{Grado de satisfacción con su propia formación profesional}

Los participantes en el estudio manifiestan que, en su curriculum formativo, existe una carencia de formación específica en temas interculturales: «[En la universidad] no te [sic] enseñan cómo compensar [los déficits del alumnado], tú tienes que aplicar tu propia metodología» (TPC28). Ante esta carencia, los futuros docentes expresan la necesidad de cursar materias optativas que los especialicen en temas interculturales: «Debería de haber una especialización [en interculturalidad], unas materias optativas en la carrera» (MIA6).

A pesar de ser conscientes de su falta de formación específica, manifiestan cierta satisfacción con su formación, en especial, desde la última reforma de los planes de estudio ${ }^{2}$. Esta reforma, entre otros cambios, transformó la diplomatura de Magisterio en el grado de Maestro en Educación, con las especialidades de Infantil y Primaria. En este contexto, los participantes distinguen entre la formación de antes y la actual: «Verdaderamente [la interculturalidad] la estudian las generaciones nuevas [de estudiantes de grado]» (MPA18). Además, estos futuros profesionales de la enseñanza estiman que ha habido una ruptura en los métodos de enseñanza.

Nosotros estamos estudiando que la interculturalidad, la diversidad, son muy importantes. Ahora mismo nos lo están inculcando, pero, ¿̇osotros creéis que a los profesores que llevan ya muchos años en la enseñanza les han inculcado esos valores? No, para nada. (TIC26)

Todos los profesores que yo he tenido nos han educado como les han educado a ellos. [En cambio,] lo que estamos haciendo ahora no se hacía hace tres años. Los profesores de Magisterio han empezado este año o hace dos con métodos que nos han hecho pensar sobre cosas importantes, como éstas [la diversidad intercultural]. (MIB9)

La percepción de los participantes no difiere de la escasa relevancia que presenta la diversidad intercultural en el currículum formativo de estos futuros profesionales de la enseñanza. En el plan de estudios, tanto el relativo al grado

2. Real Decreto 1393/2007, de 29 de octubre, por el que se establece la ordenación de las enseñanzas universitarias oficiales. 
de Educación Infantil como al grado de Educación Primaria, tan solo una asignatura de seis créditos hace referencia a la diversidad: Fundamentos Psicológicos de Atención a la Diversidad. Dicha materia enfoca la diversidad desde la perspectiva compensatoria y desde la dimensión de las necesidades educativas.

\subsection{Propuestas de mejora en el sistema educativo español relacionadas con el enfoque intercultural}

Los participantes en el estudio son conscientes de las limitaciones en sus conocimientos para abordar las situaciones interculturales de las aulas españolas. Sin embargo, son capaces de enumerar un elenco de propuestas de cambio en el sistema educativo español que está en consonancia con el enfoque intercultural. La tabla 1 resume las principales propuestas de los participantes, las cuales, a efectos de análisis, se han agrupado en subcategorías.

A continuación, se comentan brevemente estas subcategorías y sus contenidos. Primeramente, en cuanto a la transformación del enfoque curricular tradicional, una vez más, los participantes se hacen conscientes de la necesidad de formación intercultural y proponen cuatro aspectos principales que dotarían de un enfoque holístico a la educación intercultural (Aguado et al., 2008):

a) Transversalidad: «La interculturalidad se tiene que tratar transversalmente» (MPB21).

b) Naturalidad: «Creo que [la interculturalidad] no debería de ser tratada como algo especial, sino como algo natural» (MIA2).

c) Generalidad: «A los extranjeros, les favorecería que se hiciera algo común en todos los centros» (MPB24).

d) Continuidad: «La preparación [de los profesionales de la enseñanza] no puede parar nunca, debería ser continua [...] La educación tiene que crecer a la vez que la sociedad, ya que en la sociedad se están produciendo continuamente cambios» (MIB7).

Todo esto podría resumirse en el siguiente comentario:

Yo pienso que [la interculturalidad] no tiene que ver solo con el profesorado, sino con toda la sociedad, porque el profesor puede decir una cosa, pero tú $[s i c]$ llegas a tu casa y tu familia tiene ideas contrarias [...] yo creo que debería de educarse en la diversidad y la integración desde infantil, que es de donde parte todo [...] [Así, cuando el alumnado] llegue a primaria y secundaria, no habrá ningún problema. (MIA2)

Además, entre las propuestas de transformación del enfoque tradicional, los participantes incorporan al discurso la metodología de las aulas inclusivas, en consonancia con el planteamiento de otros autores (Besalú y Tort, 2009; Casanova, 2011; Gallego et al., 2012; García y Goenechea, 2011; Slee y Allan, 2001), y señalan que la única manera de conseguir centros educativos realmen- 
Tabla 1. Subcategorías de las propuestas de mejora en el sistema educativo español relacionadas con el enfoque intercultural

\begin{tabular}{|c|c|c|}
\hline & Subategorías & Propuestas de mejora \\
\hline \multicolumn{2}{|c|}{$\begin{array}{l}\text { Transformación del enfoque curricular } \\
\text { tradicional. }\end{array}$} & $\begin{array}{l}\text { - Preparar al profesorado en temas intercul- } \\
\text { turales. } \\
\text { - Adaptar el currículum formativo a las necesi- } \\
\text { dades interculturales. } \\
\text { - Aplicar metodologías innovadoras con cariz } \\
\text { intercultural. } \\
\text { - Establecer aulas inclusivas. }\end{array}$ \\
\hline \multicolumn{2}{|c|}{$\begin{array}{l}\text { Rechazo al racismo, a los estereotipos, a } \\
\text { los prejuicios y a la discriminación negativa }\end{array}$} & $\begin{array}{l}\text { - Evitar el etnocentrismo. } \\
\text { - Educar en valores no racistas: igualdad, tole- } \\
\text { rancia y respeto. } \\
\text { - Fomentar el conocimiento de otras culturas. } \\
\text { - Combatir el aislamiento y la marginación de } \\
\text { los extranjeros. }\end{array}$ \\
\hline \multicolumn{2}{|c|}{ Apoyo específico al alumnado extranjero } & $\begin{array}{l}\text { - Establecer períodos de adaptación para el } \\
\text { alumnado extranjero. } \\
\text { - Establecer clases de apoyo (idioma) para el } \\
\text { alumnado extranjero. } \\
\text { - Promover una educación individualizada (no } \\
\text { aislada) para el alumnado extranjero. } \\
\text { - Establecer tutorías grupales para el alumna- } \\
\text { do extranjero. }\end{array}$ \\
\hline & a sus familias & $\begin{array}{l}\text { - Fomentar la participación de las familias. } \\
\text { - Aumentar el apoyo a las familias. }\end{array}$ \\
\hline \multirow[t]{2}{*}{ Cooperación } & entre los diferentes agentes & $\begin{array}{l}\text { - Promover la cooperación entre la familia y la } \\
\text { escuela: talleres para familias. }\end{array}$ \\
\hline & entre el propio alumnado & $\begin{array}{l}\text { - Realizar actividades cooperativas entre el } \\
\text { alumnado. } \\
\text { - Aprender en equipo. }\end{array}$ \\
\hline \multicolumn{2}{|c|}{ Colaboración interinstitucional } & $\begin{array}{l}\text { - Implantar planes de integración que tengan } \\
\text { en cuenta la diversidad. } \\
\text { — Aumentar los programas de integración } \\
\text { social. } \\
\text { — Invertir mayor cantidad de recursos económi- } \\
\text { cos y humanos. } \\
\text { — Fomentar la laicidad en la educación: elimi- } \\
\text { nación de símbolos religiosos en el espacio } \\
\text { público. }\end{array}$ \\
\hline
\end{tabular}

Fuente: elaboración propia.

te interculturales es a través de las escuelas inclusivas: «Si queremos centros interculturales, tenemos que aplicar metodologías inclusivas» (MPB19).

La segunda subcategoría se relaciona con el rechazo del racismo, los estereotipos, los prejuicios y la discriminación negativa: «España es aún muy reacia, muy racista» (MPB22). Esto requeriría una resocialización de los futuros docentes en valores no racistas, no etnocéntricos, no estereotipados: «Las principales trabas las hemos puesto nosotros con nuestro racismo [...] el principal 
problema somos nosotros» (MIA2). De aquí puede deducirse que, si bien la formación académica es importante, no lo es menos la formación en valores: «Hay que educar para que no haya prejuicios negativos, ni tópicos [...] Es muy importante saber cómo enseñar todos esos valores a nuestros alumnos» (MPB22). También se puede deducir la importancia de un cambio de actitud, en el sentido de fomentar, entre futuros profesionales de la enseñanza, la reflexión guiada sobre sus propios conceptos, ideas y actitudes, en relación con la interculturalidad: «Pensamos que tenemos más abierta la mente de lo que realmente la tenemos [...] tenemos que cambiar nosotros» (MPB22).

La tercera subcategoría se refiere al apoyo específico al alumnado extranjero y sus familias. En cuanto a lo primero, se expresa la necesidad de: «[...] que ellos sientan que tienen tu apoyo siempre» (MIA2). Este apoyo no debe de ser exclusivo del profesorado, sino de todos los compañeros: «Si tienes una clase unida, se van a apoyar unos a otros. Las dinámicas de grupo ayudan mucho» (MPB23). Además, debería prestarse una atención diferenciada:

Una persona que no hable nuestro idioma tiene que tener una atención diferenciada. Esa persona no puede estar en la clase porque no va a entender nada. Hay que ayudarle de alguna manera, y eso es atención diferenciada [...] según las necesidades. (MPB19)

En relación con lo segundo, un tipo de apoyo específico, al que los participantes otorgan gran importancia, es el apoyo a las familias: «Hay que empezar por la familia, siendo básica la coordinación [entre la escuela y ésta]» (MIB11).

El apoyo específico al alumnado extranjero y sus familias conecta con la cuarta subcategoría: la cooperación. Aquí se resalta la necesidad de establecer cooperación entre el profesorado y las familias de inmigrantes, para una mejor educación de los hijos de éstas. En este sentido, se plantea que: «Debería de haber clases para que las familias de otras culturas puedan ayudar a sus hijos a tener éxito en nuestro sistema educativo» (MPB19). Además, se da mucha importancia al trabajo grupal: «Yo trabajaría más el aprendizaje en grupo, el aprendizaje cooperativo entre todos los alumnos, para que les sea más fácil aprender» (MIB8).

Por último, los participantes ponen de relieve la importancia de la colaboración interinstitucional. Así, los modelos holístico y sociocrítico quedan reflejados con fuerza en las propuestas de mejora que emergen en su discurso:

a) Implantar planes de integración que tengan en cuenta la diversidad: «Deberíamos de integrar a todos los extranjeros, a través de planes de integración [...] para que toda la sociedad se enriquezca» (MIA2).

b) Aumentar los programas de integración social: «Yo creo que también deben preocuparse un poco el Estado, el gobierno, con más programas de integración social» (MPB20).

c) Invertir mayor cantidad de recursos económicos y humanos: «Aunque tengamos formación, no tenemos medios, los materiales didácticos no están 
preparados para la diversidad [...] [además] sería necesario poder apoyarte en una persona que se haya especializado en diversidad» (MPB19).

d) Fomentar la laicidad en la educación: «La Iglesia debe separarse por completo de las aulas» (MPB19).

\section{Conclusiones}

En cuanto al primer objetivo de este estudio, la perspectiva dominante en el discurso de estos futuros profesionales de la enseñanza reproduce el enfoque asimilacionista y compensatorio. Llama la atención la persistencia de este tipo de enfoques en el sistema educativo español. A pesar de los avances teóricos y de la multitud de experiencias interculturales activas en el ámbito educativo, las resistencias parecen enraizarse en lugares más profundos. Dados los resultados, podría deducirse que la causa principal de tal orientación se encuentra en la escasez de conocimientos y la falta de experiencia personal en el ámbito de la diversidad intercultural de los futuros profesionales de la enseñanza; es decir, dicha perspectiva está fuertemente arraigada debido a los procesos de socialización vividos por los participantes en sus contextos socioculturales y educativos.

Que domine esta perspectiva no significa que los futuros docentes no la cuestionen. Las dudas y las contradicciones continuas, la escasez de argumentos y la demanda de formación específica abren una puerta al cambio de perspectiva hacia un enfoque intercultural, o sea, holístico y sociocrítico. En este sentido, conviene resaltar que los participantes han sido capaces de enumerar un elenco de propuestas de mejora del sistema educativo español que conectan, en gran parte, con dicho enfoque intercultural, fundamentalmente, la transformación del enfoque curricular tradicional y la cooperación entre agentes educativos.

En relación con el segundo objetivo del estudio, los participantes extraen las siguientes barreras que potencian la distancia entre teoría y práctica:

a) El desconocimiento de medidas con un claro enfoque intercultural que ya se están poniendo en práctica en el sistema educativo español.

b) Algunos de los valores personales no son consonantes con un enfoque intercultural.

c) Las ideas estereotipadas que subyacen en el consciente colectivo, aunque tales ideas empiezan a diluirse cuando se cuestionan en el plano personal e incluso desaparecen cuando se consideran propuestas de mejora.

d) La falta de una visión transversal de carácter intercultural en el currículum formativo de los futuros docentes.

A pesar de la capacidad de los participantes de visualizar tales barreras, ellos mismos son conscientes de que éstas no son fácilmente superables, dado que: «El problema principal es que la sociedad sigue sin estar preparada para asumir la diversidad» (MPA14).

Todo ello sugiere la necesidad de acortar la distancia entre la teoría y la práctica, desde una perspectiva intercultural, para mejorar en la gestión de 
la diversidad. ¿El debate se centra entonces en el cómo? En este sentido, parece lógico afirmar que la formación inicial del profesorado puede ser un recurso fundamental en este proceso de acercamiento. El debate se reabre con el proceso de renovación de los planes de estudios de grado en las titulaciones de educación infantil y primaria, tras cuatro años de andadura (el cronograma depende de la temporalización en la implantación de los grados en las distintas universidades españolas). Ante esta perspectiva, podría sugerirse que los cambios en la formación de los futuros docentes se centrasen, al menos, en cuatro ejes fundamentales:

a) Un proceso de resocialización (Besalú, 2012) en valores más cercanos al enfoque intercultural (sensibilidad, empatía, capacidad crítica, etc.).

b) Una formación centrada en la reflexión y orientada hacia conceptos, ideas y actitudes, más que en técnicas o metodologías abstractas (Windschitl, 2006).

c) El desarrollo de capacidades propias de un mundo intercultural.

d) Un prácticum docente ${ }^{3}$ que promueva un contexto apropiado a la diversidad intercultural.

Finalmente, cabe señalar que, en este proceso de cambio, el personal docente es solo un elemento más entre todos los que constituyen el sistema educativo y que, por sí solo, parece poco probable que sea capaz de modificar las prácticas educativas dominantes. La diversidad intercultural sigue siendo un reto para toda la sociedad española.

\section{Referencias bibliográficas}

Aguado, Teresa (coord.) (2006). Guía INTER: Una guía práctica para aplicar la educación intercultural en la escuela [en línea]. Madrid: Centro de Investigación y Documentación Educativa (CIDE). Entre Manos, 1. $<$ http://www.uned.es/grupointer/colentremanos001pc.pdf> [Consulta: 30 octubre 2012].

Aguado, Teresa; Gil, Inés y Mata, Patricia (2008). «El enfoque intercultural en la formación del profesorado: Dilemas y propuestas». Revista Complutense de Educación, 19 (2), 275-292.

Alegret, Juan Luís (1992). «Racismo y educación». En: Fermoso, P. (ed.). Educación intercultural: La Europa sin fronteras. Madrid: Narcea, 93-110.

Andreu, Jaime; García-Nieto, Antonio y Pérez, Ana (2007). Evolución de la Teoría Fundamentada como técnica de análisis cualitativo. Madrid: Centro de Investigaciones Sociológicas. Cuadernos Metodológicos, 40.

BANKs, James (1989). «Multicultural Education: Development, Paradigms and Goals». En: Banks, J. A. y Banks, C. A. (eds.). Multicultural Education: Issues and Perspectives. Londres: Allyn and Bacon, 2-26.

3. Asignatura de los planes de estudio de grado de Educación Infantil y Primaria (cursos segundo, tercero y cuarto). 
- (1991). «Teaching Multicultural Literacy to Teachers». Teaching Education [en línea], 4 (1), 133-144. <http://dx.doi.org/10.1080/1047621910040118>.

- (1996). Multicultural education, transformative knowledge, and action: Historical and contemporary perspectives. Nueva York: Teachers College Press.

Baquedano-López, Patricia; Alexander, Rebecca Anne y Hernández, Sera (2013). «Equity Issues in Parental and Community Involvement in Schools: What Teacher Educators Need to Know». Review of Research in Education [en línea], 37, 149-182. <http://dx.doi.org/10.3102/0091732X12459718>.

Bernstein, Basil (1986). "Una crítica a la educación compensatoria». En: Wright, C. y Foucault, M. (eds.). Materiales de sociología crítica. Madrid: Las Ediciones de la Piqueta, 203-218.

Bereiter, Carl y Englemann, Siegfried (1966). Teaching disadvantaged children in the preschool. Nueva Jersey: Prentice-Hall.

Besalú, Xavier (2012). "Interculturalidad: la resocialización del profesorado». En: López, B. y Tuts, M. (coords.). Orientaciones para la práctica de la educación intercultural: Red de escuelas interculturales [en línea]. Madrid: Wolters Kluwer España y Liga Española de la Educación y la Cultura Popular, 109-124.

<http://www.escuelasinterculturales.eu/IMG/pdf/orientacionesparalapracticadelaeducacionintercultural-2.pdf> [Consulta: 3 julio 2013].

Besalú, Xavier y Tort, Josep (2009). Escuela y sociedad multicultural: Propuestas para trabajar con el alumnado extranjero [en línea]. Madrid: Eduforma.

Bоoтн, Tony y Ainscow, Mel (2000). The Index for Inclusion: Developing Learning and Participation in Schools. Londres: CSIE.

Casanova, María Antonia (2011). Educación inclusiva: Un modelo de futuro. Madrid: Wolters Kluwer.

Colectivo IOE (2007). Inmigración, género y escuela: Exploración de los discursos del profesorado y del alumnado. Madrid: Centro de Investigación y Documentación Educativa (CIDE). Estudios Creade, 1.

Devine, Patricia G. (1989). «Stereotypes and prejudice: Their automatic and controlled components». Journal of Personality and Social Psychology [en línea], 56, 5-18. <http://dx.doi.org/10.1037/0022-3514.56.1.5>.

Dietz, Gunther y Mateos, Laura Selene (2011). Interculturalidad y educación intercultural en México: Un análisis de los discursos nacionales e internacionales en su impacto en los modelos educativos mexicanos. México: Secretaría de Educación Pública.

Gallego, Beatriz; Rodríguez, Henar y Lago, Mónica (2012). «Buenas prácticas en educación intercultural». En: López, B. y Tuts, M. (coords.). Orientaciones para la práctica de la educación intercultural: Red de escuelas interculturales [en línea]. Madrid: Wolters Kluwer España y Liga Española de la Educación y la Cultura Popular, 53-68.

<http://www.escuelasinterculturales.eu/IMG/pdf/orientacionesparalapracticadelaeducacionintercultural-2.pdf> [Consulta: 15 junio 2013].

García, José Antonio y Goenechea, Cristina (2011). «Identificando buenas prácticas interculturales desde claves inclusivas». Revista Educación y Futuro Digital [en línea]. 1. <http://www.cesdonbosco.com/revista/numeroanteriores/digital/EYFD_1.pdf> [Consulta: 2 septiembre 2013].

García, José Antonio; Sánchez, Primitivo; Moreno, Isidro y Goenechea, Cristina (2010). «Estudio del sistema y funcionamiento de las aulas de enlace de la Comu- 
nidad de Madrid: De la normativa institucional a la realidad cotidiana». Revista de Educación [en línea], 352, 473-493.

Garreta, Jordi y Llevot, Nuria (2003). El espejismo intercultural: La escuela de Cataluña ante la diversidad cultural. Madrid: Centro de Investigación y Documentación Educativa (CIDE).

Giroux, Henry A. (1992). Igualdad educativa y diferencia cultural. Barcelona: Roure.

Goenechea, Cristina; García, José Antonio y Jiménez, Rafael A. (2011). «Los dilemas de la atención educativa a los alumnos inmigrantes recién llegados: Estudio comparativo de los modelos andaluz (ATAL) y madrileño (aulas de enlace)». Profesorado: Revista de Currículum y Formación del Profesorado [en línea], 15 (3), 263-278.

Jensen, Arthur R. (1969). «How much can we boost IQ and scholastic achievement?». Harvard Educational Review, 39 (1), 1-123.

Jordán, Josep (1995). La escuela multicultural: Un reto para el profesorado. Barcelona: Paidós.

López López, María del Carmen e Hinojosa Pareja, Eva F. (2012). «El estudio de las creencias sobre la diversidad cultural como referente para la mejora de la formación docente». Educación XX1 [en línea], 15 (1), 195-218. $<$ http://dx.doi.org/10.5944/educxx1.15.1.156>.

Martínez, Asunción y Zurita, Félix (2011). «Actitudes y percepciones del alumnado de Magisterio en la especialidad de Educación Primaria ante los procesos migratorios». Aula Abierta, 49, 91-102.

Merino, José Vicente y Muñoz, Antonio (1995). «Ejes de debate y propuestas de acción para una Pedagogía Intercultural». Revista de Educación, 307, 127-162.

Ministerio de Educación, Cultura y Deporte (2013). Datos y cifras: Curso escolar 2013/2014 [en línea].

<http://www.mecd.gob.es/servicios-al-ciudadano-mecd/dms/mecd/servicios-alciudadano-mecd/estadisticas/educacion/indicadores-publicaciones-sintesis/datoscifras/Datos-y-Cifras-2013-2014-LR/Datos\%20y\%20Cifras\%202013-2014_final. pdf> [Consulta: 10 enero 2014].

MuÑoz, Antonio (1997). Educación intercultural: Teoría y práctica. Madrid: Escuela Española.

- (2001). «Hacia una educación intercultural: Enfoques y modelos». Encounters on Education, 1, 81-106.

Nieto, Sonia y Santos Rego, Miguel A. (1997). «Formación multi/intercultural del profesorado: Perspectivas en los Estados Unidos y en España». Teoría de la Educación, 9, 55-74.

Olmo, Margarita del (2012). «Buenas prácticas, ¿desde el punto de vista de quién?: Una contribución a la controversia sobre las aulas de enlace». Revista de Educación, 358, 111-128.

Osuna, Carmen (2012). «En torno a la educación intercultural: Una revisión crítica». Revista de Educación, 358, 38-58.

Sandoval, Miquel; López, Miquel; Miquel, Esther; Durán, D.; Giné, C. y Echeita, Gerardo (2002). «Index for Inclusion: Una guía para la evaluación y mejora de la educación inclusiva». Contextos Educativos, 5, 227-238.

Sandoval, Miquel; Echeita, Gerardo; Simón, Cecilia y López, Miquel (2012). Educación inclusiva: Iguales en la diversidad. Madrid: Instituto Nacional de Tecnologías Educativas y de Formación del Profesorado. Ministerio de Educación, Cultura y Deporte. 
Santos Rego, Miguel A. (1994). Teoría y práctica de la educación intercultural. Santiago de Compostela: Universidad de Santiago de Compostela. Servicio de Publicaciones e Intercambio Científico.

Slee, Roger y Allan, Julie (2001). «Excluding the included: A reconsideration of inclusive education». International Studies in Sociology of Education [en línea], 11 (2), 173-192. <http://dx.doi.org/10.1080/09620210100200073>.

Strauss, Anselm y Corbin, Juliet (1998). Basic of Qualitative Research: Techniques and Procedures for Developing Grounded Theory. 2a ed. Thousand Oaks, CA: Sage.

TERrén, Eduardo (2003). «Educación democrática y ciudadanía multicultural: El reaprendizaje de la convivencia». En: Morán, M. L. y Benedito, J. (eds.): Aprendiendo a ser ciudadanos: Experiencias sociales y construcción de la ciudadanía entre los jóvenes. Madrid: INJUVE, 259-279.

Unión General de Trabajadores (2010). Libro Blanco de la Educación Intercultural. Madrid: Secretaría para la Igualdad de la Comisión Ejecutiva Confederal de UGT y Secretaría de Políticas Sociales de la Federación de Trabajadores de la Educación (FETE-UGT).

WindsCHITL, Mark (2002). «Framing constructivism in practice as the negotiation of dilemmas: An analysis of the conceptual, pedagogical, cultural and political challenges facing teachers». Review of Educational Research [en línea], 72 (2), 131-175. <http://dx.doi.org/10.3102/00346543072002131>.

Yin, Robert K. (1989). "Case Study Research: Design and Methods». Applied Social Research Methods Series. Newbury Park, CA: Sage. 